\title{
Performance of the Pyroreflectometry in Magnetic Fusion Devices for Plasma Wall Interaction monitoring
}

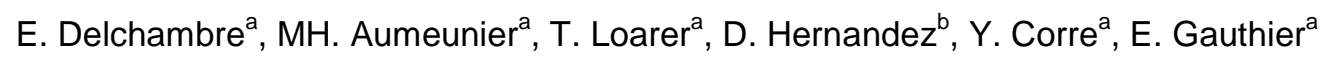 \\ ${ }^{a}$ CEA, IRFM, F-13108 Saint-Paul-lez-Durance, France \\ ${ }^{b}$ PROMES CNRS 8521, Centre Felix Trombe, BP5, Odeillo, F-66125 Font Romeu, France
}

\begin{abstract}
ITER (International Thermonuclear Experimental Reactor) is a large-scale scientific experiment that aims to demonstrate that it is possible to produce commercial energy from fusion reactions. Because of their physical and thermal properties, Beryllium and tungsten have been chosen for Plasma Facing Components to cover the interior surfaces of the vacuum vessel. Such metallic environment raises the issue related to reflecting internal surfaces which can disturb the machine protection system based on Infrared Thermography. In this paper we focus on evaluating the performance of the Pyroreflectometry method in reflective environment.
\end{abstract}

\section{1- MOTIVATIONS}

\subsection{Magnetic Fusion}

Fusion is the source of energy in the sun and other stars. A star starts to shine when, under the force of gravity, the matter in its very heart attains sufficiently high densities and temperatures to set off thermonuclear reactions, which then release energy. On Earth, it is possible to reproduce these reactions by confining and maintaining plasma at very high temperature; plasma is the fourth state of the matter where electrons are completely detached from the nucleus. This plasma is confined in a torus-shaped vacuum vessel, also called tokamak, created by magnetic fields, described as magnetic confinement. Although different isotopes of light elements can be paired to achieve fusion, the deuterium-tritium (D-T) reaction has been identified as the most efficient for fusion devices.

$$
\begin{gathered}
\mathrm{D}+\mathrm{T} \rightarrow{ }^{4} \mathrm{He}(3.5 \mathrm{MeV})+\mathrm{n}(14.1 \mathrm{MeV}) \\
{ }^{6} \mathrm{Li}+\mathrm{n} \rightarrow{ }^{4} \mathrm{He}+\mathrm{T}
\end{gathered}
$$

It is on this reaction that research on controlled fusion is conducted. The temperatures required for thermonuclear fusion is greater than a hundred million degrees and the density is very low $\sim 10^{-5} \mathrm{x}$ the air density. The challenge to perform at steady state operation is to exhaust energy created (14.1MeV from neutrons) and ashes (He) without perturbing the core plasma performance. Such constraints explain the importance of the plasma facing components design as well as the crucial need of reliable temperature monitoring.

Using tritium and deuterium as fuel has some interesting consequences, for example there is no possibility of chain reaction, and the fuel has to be fed into the plasma continuously in very small precise amounts, in a similar way to a gas burner. The total amount of fuel confined by the plasma at any moment is only sufficient for a few seconds burn if the supply of fuel is terminated. Deuterium can be found in sea water $\left(33 \mathrm{gr} / \mathrm{m}^{3}\right)$ which is sufficient for billions of years. Tritium is a fast-decaying radioelement of hydrogen which occurs only in trace quantities in nature. It can be produced during the fusion reaction, when neutrons escaping the plasma interact with lithium contained in the blanket wall of the tokamak. Lithium is plentiful in the Earth's crust and the known reserves of lithium would last for at least one thousand years. 


\subsection{Toward Fusion power plant}

Research on controlled fusion has made great strides over the last few years both in Europe and in the world, whether in physics, materials, technology or in the concept of a future fusion reactor. This progress is the result of work on many "specialised" experimental installations. The JET tokamak, for example, is dedicated to the physics of high performance plasmas over short periods (a few seconds). The Tore Supra machine is more specialized on lower performance plasmas that last much longer (6 minutes). The Fusion research community involved in these studies is ready to take a further step: demonstrate the sustained combustion of deuterium-tritium plasma over long periods (400 seconds). This is the main goal of ITER (International Thermonuclear Experimental Reactor), the next generation machine. The information, technologies and experience provided by ITER will be crucial to the development of a demonstration power plant DEMO as shown in figure 1. DEMO will be the next experimental device to follow ITER, and predecessor to a commercial-sized fusion reactor. DEMO would generate electricity at the level of a few hundred MW and utilize all technologies necessary for a commercial device.

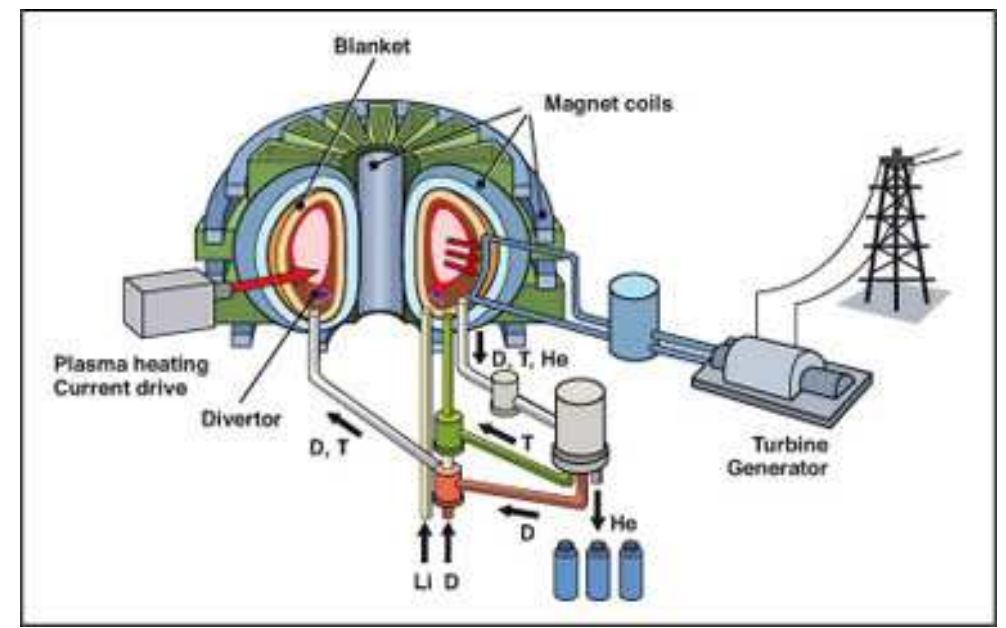

Figure 1: Cutaway view of the ITER tokamak, with the fusion plasma shown in purple.

ITER has been designed to produce $500 \mathrm{MW}$ of output power for $50 \mathrm{MW}$ of input power, or ten times the amount of energy put in. The size of the vacuum vessel dictates the volume of the fusion plasma; the larger the vessel, the greater the amount of power that can be produced. The ITER vacuum vessel will be twice as large and sixteen times as heavy as any previous tokamak, with an internal diameter of 6 meters. It will measure a little over 19 meters across by 11 meters high, and weigh in excess of 5,000 tons.

The blanket covers the interior surfaces of the vacuum vessel, providing shielding to the vessel and the superconducting magnets from the heat and neutron fluxes of the fusion reaction. The neutrons are slowed down in the blanket where their kinetic energy is transformed into heat energy and collected by the coolants. In a fusion power plant, this energy will be used for electrical power production. The figure 2 shows a cut-away of the ITER vacuum vessel showing part of the inner wall made of beryllium and the Divertor made of tungsten at the bottom. 


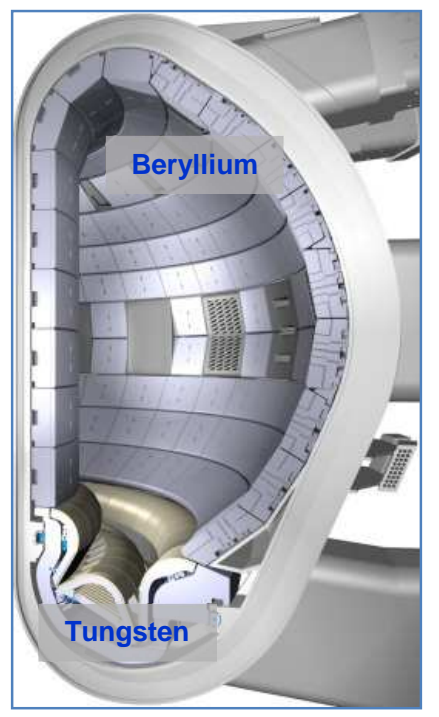

figure 2: Cut-away of the ITER Vacuum Vessel showing part of the inner wall made of beryllium and the Divertor made of tungsten at the bottom.

\subsection{Thermal loads}

The ITER blanket is one of the most critical and technically challenging components in ITER: together with the divertor it directly faces the hot plasma. The divertor is one of the key components of the ITER machine. Situated along the bottom of the vacuum vessel, its function is to extract heat and helium ashes, acting like a giant exhaust system. Plasma facing components will be exposed to intense thermal loads. For the first wall these thermal loads will remain in general below $1-5 \mathrm{MW} \cdot \mathrm{m}^{-2}$ during steady state operation; however, special attention has to be paid to high heat flux components like the divertor. This component have to withstand power densities up to approximately $20 \mathrm{MW} \cdot \mathrm{m}^{-2}$ during steady state plasma operation and in addition to these quasi-stationary loading conditions, the plasma facing components may be exposed to several types of short transient thermal pulses: i) disruptions: short interruptions of the plasma current leading to a local deposition of high energies up to $30 \mathrm{MJm}^{-2}$ within a few milliseconds j) vertical displacement events (VDEs): spatial instabilities of the plasma in which the first wall is hit by the plasma with $350 \mathrm{MJ}$ for up to $200 \mathrm{~ms} \mathrm{k}$ ) ELMs: cyclic energy depositions of about $1 \mathrm{GWm}^{-2}$ over $500 \mu \mathrm{s}$. All these transients events with high local energy depositions which may lead to surface melting, evaporation or cracking and finally to an erosion of the plasma facing components.

\subsection{Materials}

The choice of the surface material for the divertor is an important one. The plasma facing components situated on the divertor are situated at the intersection of magnetic field lines where the high-energy plasma particles strike the components. Their kinetic energy is transformed into heat; the heat flux received by these components is extremely intense and in order to obtain steady state the components need to be actively cooled. Only very few materials are able to withstand high heat flux leading to surface temperatures of up to $3000{ }^{\circ} \mathrm{C}$ for the projected 20-year lifetime of the ITER machine. Tungsten, which is a high-refractory material, has been chosen due to its extremely high melting point, which offers the advantage of a long lifetime. Beryllium has been chosen as the element to cover the first wall. Due to its affinity to oxygen it is an excellent getter material which guarantees plasma discharges with low impurity levels and is also characterized by a rather good thermal conductivity. The rest of the blanket shield will be made of high-strength copper and stainless steel. 


\subsection{Infrared diagnostic}

In order to provide a reliable and safe operation, two approaches are required: on one hand a plasma scenario compatible with heat loads limits, enable to reduce the power load during stationary phase and to mitigate transient events has to be ran. One the other hand the accuracy in determining the surface temperature is of a main interest and a reliable monitoring of the temperature by InfraRed (IR) Thermography is required. ITER will have wide angle viewing systems and a divertor thermography diagnostic, which shall provide infrared coverage of the divertor and large parts of the first wall surfaces with spatial and temporal resolution adequate for operational purposes and higher resolved details of the divertor and other areas for physics investigations [1].

In early fusion study, most of the machines were equipped with carbon type materials (rather absorbing materials with an emissivity 0.9), a material that presents the advantage of high thermal conductivity, easy to manufactured and affordable. The 2D monitoring of the surface temperature using IR cameras is very well handled in fusion community, who has developed an experience on real time feedback control of long plasma pulses, and on safety control of heat flux limits of actively cooled components [2]. However, long discharges have raised the issue of lifetime components due to chemical and physical erosion [3] and therefore the growth of dust and carbon deposits [4] bringing up a tritium retention issue [5] which can be potentially a serious constraints for operation [6]. As a consequence carbon has been replaced by tungsten and beryllium for future fusion devices. This new environment with full metallic surfaces and low emissivity $(\sim 0.2-0.4)$ raises the issue of surface temperature measurement in reflective environment. Indeed low and changing emissivity of the PFCs can lead to an underestimation of temperature and PFC damage; high reflectivity can lead to overestimations and premature protections action. As a matter of fact, the infrared radiance measured by standard pyrometry can be dominated by the reflected flux even if the surrounding temperature is lower than the temperature of the target [7]. In the case of high reflective flux, the technique can be coupled with active pyrometry like photo-thermal method [8] [9]. In this method the emitted flux is de-correlated from the reflective flux by modulating the surface temperature with pulsed or modulated laser. Since two measurements at two different wavelengths are required, this technic is similar to a bi-color pyrometry method but the emissivity ratio is still required to extrapolate the true temperature of the surface. Another method, able to determine simultaneously the true temperature and the emissivity, has been developed at CNRS-PROMES [10]. This method is called pyroreflectometry but the performances in a high reflective environment have not been experimentally evaluated yet. The aim of this task is to determine the application range of pyroreflectometry and the developments required to be applicable in Fusion devices environment.

\section{2- Pyroreflectometry principle}

The pyroreflectometry is a punctual technic which allow to measure simultaneously the true temperature and the emissivity of an opaque surface. The method has been developed at the CNRS-PROMES at two wavelengths. The principle of the pyroreflectometry is to get the true surface temperature of metallic target (with $\varepsilon \neq 1$ ) by measuring both the bidirectional reflectivity, $\rho$ ", and the temperature radiance, $T_{R}$, at two wavelengths. The bidirectional reflectivity, $\rho "$ is measured from pulsed laser diodes working at $\lambda_{1}=1.3 \mu \mathrm{m}$ and $\lambda_{2}=1.55 \mu \mathrm{m}$. The radiance temperature, $T_{R}$, is deduced directly from the total flux emitted by the target and collected by the photodiode by using the Planck's law and a calibration of the apparatus with a blackbody.

The bicolor pyroreflectometry is not based on standard hypothesis such as Lambertian sources or grey body. Indeed the method introduces the diffusion factor $\eta$ (the ratio of the hemispherical reflectivity $\rho$, $\cap$ to the bidirectional reflectivity $\rho "$ ". $\pi$ corresponding to illumination direction of the target with a laser and the observation direction of the pyroreflectometer). The main hypothesis is to consider that the diffusion factor is independent 
with $\lambda_{1}$ and $\lambda_{2}$. This key parameter is a thermo-optical property directly linked to the surface state: when $\eta$ converges to 1 , the surface is Lambertian ( $\rho$ " is constant for any incident and viewing direction); when $\eta$ converges to 0 , the surface is specular ( $\rho$ " is strongly dependant on the incident and viewing direction). The invariance of $\eta$ with wavelengths of measurement has been experimentally validated at the solar furnace with thermo-couple for temperature validation and multi-directional reflectrometer on several metallic surfaces [11]. By introducing this key parameter, the advantage is to express the emissivity as a function of the diffusion factor: $\varepsilon=$ $1-\rho^{\prime \cap}=1-$ - .n. $\rho "$. The measured radiance can be written has following for each wavelength:

$$
R(T, \lambda)=\left(1-\pi \cdot \eta(T) \cdot \rho^{\prime \prime}(T, \lambda)\right) \cdot R(T, \lambda)
$$

The Wien law is used in our case since this approximation is true as soon as $\lambda T \ll 14400 \mu \mathrm{mK}$. The $T$ as well as $\eta$ can be then deduced by solving a system of two equations, respectively for $\lambda_{1}$ and $\lambda_{2}$ with two unknown parameters $(T$ and $\eta)$ :

$$
\begin{aligned}
& 1 / T=1 / T_{R \lambda_{1}}+\frac{\lambda_{1}}{C_{2}} \ln \left(\varepsilon_{\lambda 1}\right)=1 / T_{R \lambda_{1}}+\frac{\lambda_{1}}{C_{2}} \ln \left(1-\pi \cdot \eta(T) \cdot \rho^{\prime \prime}\left(T, \lambda_{1}\right)\right) \\
& 1 / T=1 / T_{R \lambda_{2}}+\frac{\lambda_{2}}{C_{2}} \ln \left(\varepsilon_{\lambda 2}\right)=1 / T_{R \lambda_{2}}+\frac{\lambda_{2}}{C_{2}} \ln \left(1-\pi \cdot \eta(T) \cdot \rho^{\prime \prime}\left(T, \lambda_{2}\right)\right)
\end{aligned}
$$

$\mathrm{C}_{2}=$ (h.c)/ $\mathrm{k}_{\mathrm{B}}$ where $\mathrm{h}$ is the Planck constant, $\mathrm{c}$ is the speed of light and $\mathrm{k}_{\mathrm{B}}$ is the Boltzman constant.

The solution converges to $T^{*}=T$ and $\eta$.

In order to study the sensitivity of the method on bidirectional reflectivity measurement, the error calculation has been evaluated from (4) and gives the relation:

$$
\frac{\Delta T^{*}}{T^{*}}=\frac{T^{*} \Delta T_{R, \lambda}}{T_{R, \lambda}^{2}}+\frac{\pi \lambda T^{*}}{C_{2} \varepsilon}\left[\eta . \Delta \rho^{\prime \prime}+\rho^{\prime \prime} . \Delta \eta\right](6)
$$

In previous analysis [12] we have concluded that the technic is sensitive to the reflectivity measurement and the accuracy on T increases when the measurement of the bidirectional reflectivity, $\rho$ ", is optimum. Since tokamak environment implies a remote measurement up to $\sim 10 \mathrm{~m}$ and requires specific optical development which can potentially reduce the transmission of the optical chain, the power of the diode lasers, which are currently 80 $\mathrm{mW}$ needs to be amplified in order to keep a signal above the detection threshold for better bidirectional reflectivity measurement. The choice of the working wavelength range is also of a main interest. Indeed, on the contrary to the standard bicolour thermography and/or active pyrometry where $\Delta \lambda\left(\lambda_{2}-\lambda_{1}\right)$ has to be maximised to reduce the uncertainty on the surface temperature [13], the error on temperature with the pyroreflectometry method depends on the choice of $\lambda_{1}$ and $\lambda_{2}$ (see equ. 6). It is worth noting that the choice of wavelength was initially "due to their proximity (for diffusion factor invariance), to the silica optical fibres and the availability of industrial components of low cost in the spectral range" [14]. However the choice of wavelength range can be modified and the choice of wavelengths (in order to keep the method applicable) has to take into account, in priority, the bi-directional reflectivity of the material. Indeed the equation system can be solved only when the bidirectional reflectivity is different at both wavelengths in particular when $\rho^{\prime \prime}{ }_{1}<\rho^{\prime \prime} \wedge_{2}(\lambda 1<\lambda 2)$ where mathematically there is only one solution (which is observed on most of the metallic surfaces) [15]. When $\rho$ " ${ }_{11}>\rho{ }^{\prime \prime}{ }_{k 2}$, two calculated solutions can be obtained. Thus, it is necessary to use a third wavelength to determine the convergence to a single point. When $\rho " \lambda_{1}=\rho{ }^{\prime \prime}$, the surface is a grey body and the surface temperature can be calculated with the color temperature, $T_{C}$, deduced from the standard bi-color pyrometry method $\left(T_{C}=\left(1 / \Lambda_{1}-\right.\right.$ $\left.\left.1 / \lambda_{2}\right) /\left(1 / \lambda_{1} T_{1}-1 / \lambda_{2} T_{2}\right)\right)$. 


\section{3- Effect of Parasitic reflection}

The pyroreflectometry has been developed and validated on metallic surfaces (with $\varepsilon \neq 1$ ) in non-reflective environment. The use of metallic plasma facing components in future fusion devices implies that the flux collected by the camera will include also a so called parasitic flux coming from the multiple reflections. We have been first interested to determine the impact of this parasitic reflection on the performances of the pyroreflectometry method (section 3) and we will discuss how to manage with it (section 5).

In order to numerically simulate a reflection in the pyroreflectometry method, the temperature luminance measured at each wavelength is modified by adding a reflection term modelled by a single Planck emission where $T_{i}$ is considered as a global surrounding temperature. The total radiance measured by the detector can be written as following:

$$
R\left(T_{R}, \lambda\right)=\left(1-\pi \cdot \eta(T) \rho^{\prime \prime}(T, \lambda)\right) \cdot R(T, \lambda)+\pi \cdot \eta(T) \cdot \rho^{\prime \prime}(T, \lambda) \cdot R\left(T_{i}, \lambda\right)
$$

For this first numerical evaluation, we assumed that the weighted sum of all parasitic sources in the environment can be modelled as a single radiance emitted from the whole surrounding surfaces like in an integrated sphere which is traduced by multiplying $R(T i)$ by the hemispherical reflectivity $\rho^{n \prime}=\pi . \eta$. $\rho "$.'

The bidirectional reflectivity measurement is not disturbed by the reflected flux because the bidirectional reflectivity is deduced from the emitted signal subtracted to the emitted and reflected (laser diode) emission [10] and since the parasitic signal appears in each term, the bidirectional reflectivity measurement do not depends on $\mathrm{Ti}$. As a consequence the radiance temperature, $T_{R}$, is directly calculated by inverting the Planck function from the relation (5) and then introduced in the first terms of the linear equations system (2 \& 3). In order to evaluate the performance of the pyroreflectometry on tungsten surface, the following numerical assessments uses experimental data as an input in the simulation with: diffusion factor, $\eta=0.877$, bidirectional reflectivity $\rho "=0.256$ $\mathrm{sr}^{-1}$ and $0.278 \mathrm{sr}^{-1}$ respectively at $\lambda_{1}=1.3 \mu \mathrm{m}$ and $\lambda_{2}=1.55 \mu \mathrm{m}$. Indeed the technique has been tested on one of the European fusion devices for in-situ validation (remote measurement, laser alignment) on a tungsten target introduced during plasma discharge [16].

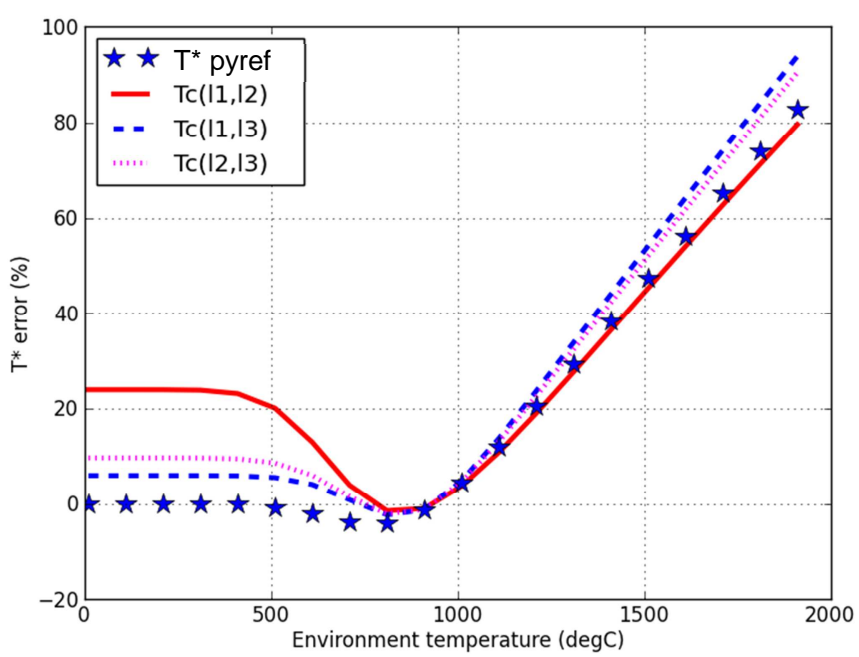

Figure 2: Effect of parasitic reflection on color temperature $T_{C}$ and recovered temperature $T^{\star}$ calculated by the pyroreflectometry method simulated on a tungsten surface at $T=938 \mathrm{C}$ and diffusion factor $\eta=0.876 . \lambda_{1}=1.3 \mu \mathrm{m}$, $\lambda_{2}=1.55 \mu \mathrm{m}$ and $\lambda_{3}=0.84 \mu \mathrm{m}$.

Figure 2 shows the error on $T^{*},\left(T^{*}-T\right)^{*} 100 / T_{\text {TRUE, }}$ the convergence temperature deduced from the pyroreflectometry as a function of the global surrounding temperature, $\mathrm{Ti}$, which varies from $400^{\circ} \mathrm{C}$ up to $1900{ }^{\circ} \mathrm{C}$ 
with $T=938^{\circ} \mathrm{C}$. For comparison, the color temperatu re, $T_{C}$, deduced from the standard bi-color pyrometry method is also plotted (assuming grey body with an emissivity ratio equal to one) using three wavelengths in order to highlight the effect of gap between wavelengths, $\Delta \lambda=\lambda_{2}-\lambda_{1}$.

We can distinguish three different cases:

1. $\mathrm{Ti}=\mathrm{T}=938^{\circ} \mathrm{C}$ : The color temperature $\mathrm{T}_{\mathrm{C}}$ as well as the temperature deduced from the pyroreflectometry, $T^{*}$, is equivalent to the true temperature. This configuration is equivalent to a measurement through an integrated sphere.

2. $\mathrm{Ti}>\mathrm{T}$ : The surface temperature is over-estimated up to $60 \%$ when $\mathrm{Ti}=1600{ }^{\circ} \mathrm{C}$, either for $\mathrm{T}_{\mathrm{C}}$ or $\mathrm{T}^{*}$.

3. $\mathrm{Ti}<\mathrm{T}$ : The color temperature over-estimates the true temperature of the surface temperature up to $23 \%$ and the over-estimation increases when $\Delta \lambda$ decreases. The temperature $\mathrm{T}^{*}$ deduced from the pyroreflectometry method gives a better results with an under-estimation $<5 \%$.

Similar simulation has been made with a true temperature at $1600{ }^{\circ} \mathrm{C}$. In this case, the temperature is underestimated of about $\sim 1 \%$ and $\sim 2 \%$ respectively for $T_{i}=800^{\circ} \mathrm{C}$ and $1100{ }^{\circ} \mathrm{C}$. These results highlight the particularity of the pyroreflectometry which introduces in the measured radiance (emitted + reflected) (5), a parameter independent with the wavelength. Moreover the radiance temperatures, $T_{R}$, assumes an emissivity equal to one which reduces the reflected contribution at each wavelength which is favourable in the case $T i<T$ and shows the asset of the pyroreflectometry compared to bicolor pyrometry.

Since the pyroreflectometry is not influenced by the environment when viewing the hottest surfaces, this method is demonstrated to be very well adapted when observing the hottest surface in a high reflective environment like in ITER where the maximum heat flux is expected, such as on the divertor. The main interest of this method is to be capable of providing the temperature of the hottest components independently to the emissivity knowledge which is a significant asset for a safety system compared to the standard bicolour pyrometry which is more disturbed by the surrounding temperature. Due to the near infrared range and the fixed integration time due to the use of photodiode detector, the temperature detection threshold is limited to $\sim 500{ }^{\circ} \mathrm{C}$. Initially the method should have been developed in the mid infrared range in order to be compatible with the 3-5 $\mu \mathrm{m}$ IR cameras routinely used in the fusion community. The idea was to develop a $2 \mathrm{D}$ pyroreflectometry ideally using bicolor detector in order to get both temporal and spatial coherence. Unfortunately laser emitting in this wavelength range are available either as compact quantum cascade laser semiconductor prototypes (which raises the issue or stability and reproducibility) or massive and expensive industrial laser sources. That is the reason why it was decided to develop in a first step a 2D pyroreflectometer adapted to fusion needs in the near infrared range.

\section{4- Towards 2D Pyroreflectometry}

Plasma components have to withstand quasi-stationary power densities up to approximately $20 \mathrm{MW} \cdot \mathrm{m}^{-2}$ as well as several types of short transient thermal pulses within a few hundreds of micro and milliseconds. Such fast transient events can have asymmetric power loads [17] with filamentary structure [18]. That is the reason why infrared coverage of the divertor and large parts of the first wall surfaces shall be provided in ITER with specifications of about $\sim 3 \mathrm{~mm}$ for spatial resolution, $10 \%$ accuracy for surface temperature and $0.02 \mathrm{~ms}$ for temporal resolution [19]. As a matter of fact the current punctual bicolor pyroreflectometry technic needs to be extend to a 2D mapping in order to be applicable for fusion application. A 2D development has been achieved at the University of Toulouse (Mines-Albi) [20]. The transposition from a punctual technique to a matricial detector (camera) is called thermoreflectometry. These measurements are achieved with a modified InGaAs with a spectral response from $0.4 \mu \mathrm{m}$ to $1.7 \mu \mathrm{m}$ coupled with two laser sources at $1.3 \mu \mathrm{m}$ and $1.55 \mu \mathrm{m}$. For an opaque material with a diffuse surface, the technique allows the bidirectional reflectivity to be tracked on line and the true 
temperature to be determined without the emissivity knowledge. However the current system is slowed down by the use of a filter wheel and allows 1 image every 30s which limits its application to stationary phenomenon. In order to consider a development dedicated to tokamak environment, the development of 2D pyroreflectometry has to take into account: fast transient heat loads and reflectivity measurement throw absorptive optical chain (window, beam splitters, mirrors, lenses, filters). In order to follow fast transient phenomenon (within a few milliseconds), we need to eliminate the slowing issue due to the use of a filter wheel by using a multiple detectors system. Results obtained by R. Gilblas [20] with thermoreflectometry highlighted that the measurement is sensitive to the solid angle of the device especially for high reflective surface. For this reason a multi-spectral system has to be coupled with an optical system allowing spatial coherence observing at different centred wavelengths the same surface from identical solid angle. The solution proposed here is the use of dichroic filters (allowing better transmission $\sim 90 \%$ than beam splitter 50\%) coupled with band-pass filters. A schematic view of such system is shown in figure 3 .

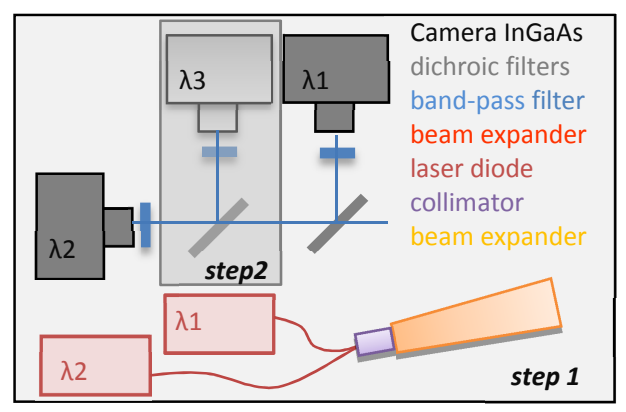

Figure 3: schematic view of the 2D pyroreflectometry experimental concept.

The experimental concept is based on a simultaneous observation of a target from the same solid angle at two wavelengths, and later on three wavelengths (step 2) in order to 1) study the effect of wavelength choice 2) enable the equation system to be solved (when the bi-directional reflectivity is different at both wavelengths where mathematically there is only one solution by solving a 3 linear equations system with two unknown parameters 3 ) test the tricolor pyroreflectometry approach which will be discussed in the following section. The aim of the work is to develop a prototype in laboratory and to test it on reflective surface. Parasitic reflection will be simulated by facing two blackbodies: the first one to heat up a metallic target (emissivity in the range of 0.2-0.6), the second one to simulate a parasitic source.

\section{5- Towards tricolor pyroreflectometry to manage reflections disturbance.}

In this section, we study the feasibility to use a third wavelength to compensate the parasitic reflection. Indeed, the introduction of a third wavelength allows to solve a system of three non-linear equations (equation 6) with three unknown parameters ( $T, \eta$ and the environment temperature, $T_{i}$ ). The equation (6) is deduced from the equation (5), which takes into account a reflective term.

It is worth noting that this new concept is different from the tricolor pyroreflectometry mentioned in 2005 by D. Hernandez [21] which is required for certain material where the condition $\rho " \lambda_{1}<\rho "{ }_{\lambda 2}$ is not verified. Indeed as mentioned in section 2 , the equation system (2\&3) can be solved only when the bi-directional reflectivity is different at both wavelengths in particular when $\rho " \lambda_{1}<\rho " \lambda_{2}(\lambda 1<\lambda 2)$ where mathematically there is only one solution. Thus, it is necessary to use a third wavelength to determine the convergence to a single point. This implies to solve a system of three equations with two unknown parameters ( $T$ and $\eta$ ) which is completely different 
to the tricolor pyroreflectometry mentioned in this paper which consists in solving a system of three equations and three parameters $(\mathrm{T}, \mathrm{n}$ and $\mathrm{Ti})$.

$$
\frac{1}{T}=\frac{\lambda}{C_{2}}\left[\ln \left(1-\pi \eta \rho^{\prime \prime}\right)-\operatorname{Ln}\left[\exp \frac{-C_{2}}{\lambda T_{R \lambda}}-\pi \eta \rho^{\prime \prime} \exp \frac{-C_{2}}{\lambda T_{i}}\right]\right] \text { (6) }
$$

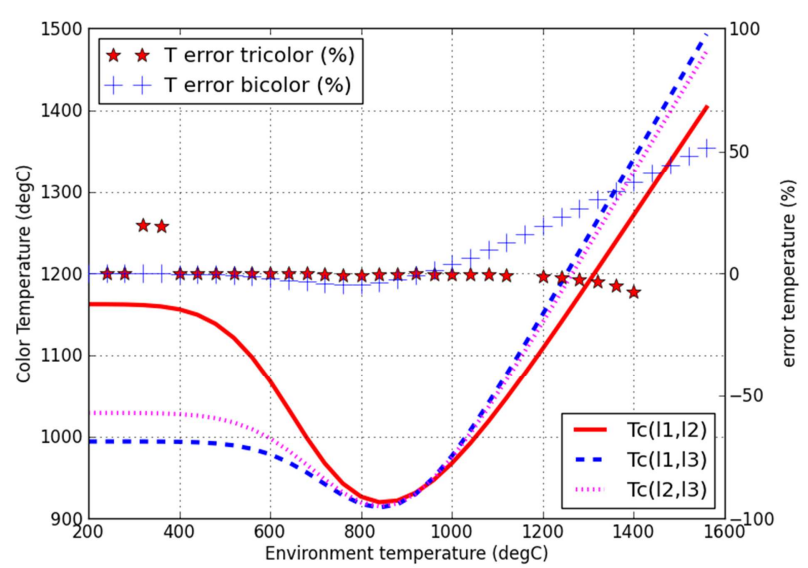

Figure 4: comparison of bicolor and tricolor pyroreflectometry simulating a reflective environment.

This tricolor approach in a reflective environment has been numerically tested taking $\lambda_{3}=840 \mathrm{~nm}$, the third wavelength used by D. Hernandez [21] and assuming a linear tungsten reflectivity [22] which implies a reflectivity of about $\rho " \lambda_{3}=0.229 \mathrm{sr}^{-1}$. For this first numerical evaluation, we assumed that the weighted sum of all parasitic sources in the environment can be modelled as a single Planck emission. The hypothesis has to be checked by using a Monte Carlo ray tracing software [7] able to take into account all multiple reflections within the vessel.

A relative good convergence is obtained when varying $T_{i}$ from $400{ }^{\circ} \mathrm{C}$ to $1400 \mathrm{C}$ as shown in figure 4 . This calculation shows that in this particular case of tungsten with a parasitic source modelled as a single luminance emitted like in an integrated sphere and assuming a linear reflectivity, the non-linear equations system converges to a true temperature even when $\mathrm{Ti}>\mathrm{T}$. Nevertheless this preliminary work is on-going and tests on algorithms stability are needed. The assumptions made on the surrounding temperature as well as the reflectivity value have also to be evaluated and tested as well as the choice of wavelength.

\section{7- Conclusions and perspectives}

The Pyroreflectometry is applicable on metallic surfaces (with low emissivity) and can provide surface temperature of the hottest component in fusion devices. The main asset of the pyroreflectometry method compared to bicolor pyrometry technique which measures the color temperature is that to be capable of measuring hottest surface temperature independently to the emissivity knowledge. This result highlights the possibility for the pyroreflectometry to measure the surface temperature of components expected to receive the highest heat load such as the divertor target in ITER. Moreover, since the bi-directional reflectivity measurement is not disturbed by the reflected flux, the method can still provide a thermo optical property linked to the surface state independently from the surrounding thermal behaviour. The extension of the punctual method to $2 \mathrm{D}$ is currently on-going at CEA-Cadarache in order to fulfil the Fusion operation constraints. The introduction of a third wavelength is currently investigated numerically and experimentally, and could allow to extend the application domain to high reflective environment $\left(T_{i}>T\right)$. In order to mitigate the impact of the reflections in complex 
environment like in ITER, the strategy is on one hand to characterize optical properties like the reflectivity to implement it to $3 \mathrm{D}$ ray-tracing code simulations and on the other hand to develop new generation of IR diagnostics enable to cope with reflections issue like photo-thermal method coupled with pyroreflectometry.

\section{Aknowledgments}

This work, supported by the European Communities under the contract of Association between EURATOM and CEA, was carried out within the framework of the European Fusion Development Agreement. The views and opinions expressed herein do not necessarily reflect those of the European Commission.

\section{References:}

[1] R. Reichle et al., Rev. Sci. Instrum. 81, 10E135 (2010)

[2] D. Guilhem Fusion Engineering and Design 74 (2005) 879-883

[3] E. Dufour et al., 32nd EPS Conference on Plasma Phys. Tarragona, 27 June - 1 July 2005 ECA Vol.29C, P5.002 (2005)

[4] E. Delchambre et al., Journal of Nuclear Materials 337-339 (2005) 1069-1072

[5] T. Loarer et al., Journal of Nuclear Materials, 390-391 (2009) 20-28.

[6] Federici, Journal of Nuclear Materials 266-269 (1999) 14-29.

[7] M.H. Aumeunier et al., Rev. Sci. Instrument, Vol 81, 10 E524 (2010)

[8] T. Loarer, Applied Optics 31(25)1992 5350

[9] S. Amiel et al., submitted to the 6th European Thermal Sciences Conference Eurotherm 2012

[10] D. Hernandez et al., Rev. Sci. Instrum. 66, 5548 (1995)

[11] D. Hernandez et al., Rev. Sci. Instr. 80, 094903 (2009)

[12] E. Delchambre et al., Phys. Scr. T145 (2011) 014078 (4pp)

[13] T. Loarer, Contrib. Plasma Phys. 51, No. 2-3, 201-206 (2011)

[14] D. Hernandez et al., Measurements 42 (2009) 836-843

[15] D. Hernandez et al., Rev. Of Sci. Instr. 76, 024904 (2005)

[16] Report Contract CEA and PROMES-CNRS. Ref: V3448.001-4

[17] T. Eich et al., Phys. Rev. Lett. 91, 195003 (2003).

[18] A. Kirk et al., Phys. Rev. Lett. 92, 245002 (2004)

[19] R. Reichle IR thermography for ITER

[20] R. Gilblas, 10th edition of the Quantitative Infrared Thermography, p.105-112, Québec City, Canada, July 2730, 2010.

[21] D. Hernandez et al., REVIEW OF SCIENTIFIC INSTRUMENTS 76, 024904 s2005d

[22] J. Gardner et al., J. Phys. E: Sci. Instrum., Vol. 13, 1980 